\section{Cahiers de Narratologie}

Analyse et théorie narratives

13 | 2006

Nouvelles approches de l'intertextualité

\title{
De l'intertextualité à la récriture
}

\section{Anne-Claire Gignoux}

\section{OpenEdition}

Journals

Édition électronique

URL : http://journals.openedition.org/narratologie/329

DOI : 10.4000/narratologie.329

ISSN : 1765-307X

Éditeur

LIRCES

\section{Référence électronique}

Anne-Claire Gignoux, « De l'intertextualité à la récriture », Cahiers de Narratologie [En ligne], 13| 2006, mis en ligne le 25 septembre 2016, consulté le 10 décembre 2020. URL : http://

journals.openedition.org/narratologie/329; DOI : https://doi.org/10.4000/narratologie.329

Ce document a été généré automatiquement le 10 décembre 2020.

\section{(c) (i) $(9)$}

Cahiers de Narratologie - Analyse et théorie narratives est mis à disposition selon les termes de la licence Creative Commons Attribution - Pas d'Utilisation Commerciale - Pas de Modification 4.0

International. 


\title{
De l'intertextualité à la récriture
}

\author{
Anne-Claire Gignoux
}

1 Les critiques qui ont retracé l'historique du concept d'intertextualité ${ }^{1}$ ont toujours souligné le flou terminologique et la multiplicité des termes du métalangage en concurrence; on parle ainsi de dialogisme, d'intertextualité, d'hypertextualité ou de récriture. Ce qui est plus grave, c'est que ce flou terminologique traduit une véritable confusion entre des pratiques différentes, et entraîne un manque de rigueur dans les études ou du moins dans la classification des différents phénomènes. Dans les limites de cet article, nous nous sommes attachée à éclairer un seul de ces phénomènes: la récriture. Dans le domaine de la stylistique, nous avons besoin d'un concept opératoire, solide, formellement isolable sur des éléments de la chaîne syntagmatique; c'est pourquoi nous avons voulu définir précisément le concept de récriture en le confrontant à celui d'intertextualité, en le situant dans ce vaste domaine.

\section{Problèmes de définition de l'intertextualité}

2 On date l'apparition du terme «intertextualité » de 1967. Les pionniers de l'intertextualité que sont Mikhail Bakhtine, Julia Kristeva, Laurent Jenny, Michael Riffaterre et Gérard Genette, sont des théoriciens venus d'horizons très divers. Dès sa naissance, le dialogisme défini par Bakhtine est constitué de deux facettes. Il est, d'une part, inhérent à l'écriture : tout énoncé, par sa dimension linguistique, renvoie à d'autres textes. Un énoncé ne peut pas ne pas être intertextuel. Tout mot a déjà servi. D'autre part, il appartient à telle ou telle esthétique, et procède, dans la Satire Ménippée ou dans le roman, d'un choix formel. Cette bivalence ne trouve pas vraiment de résolution chez les successeurs de Bakhtine. En 1969, J. Kristeva pose les fondements de l'intertextualité : « le mot (le texte) est un croisement de mots (de textes) où on lit au moins un autre mot (texte) $»^{2}$. Avec l'article de L. Jenny publié dans Poétique en $1976^{3}$, et qui fait date, on s'éloigne un peu plus du champ de la philosophie pour entrer véritablement dans la pratique textuelle. L. Jenny propose ainsi un modèle d'interprétation poétique de l'intertextualité. M. Riffaterre explore depuis la fin des années 70 sa théorie de 
l'intertextualité, dans le cadre d'une théorie de la réception. Enfin, dans son livre paru en 1982, Palimpsestes ${ }^{4}$, G. Genette s'attache avec exhaustivité à étudier tous les faits d'inter textualité, qu'il a rebaptisée du nom plus large de transtextualité. La poétique, selon lui, ne doit pas se borner au texte, mais étudier la transtextualité.

3 De cette multiplicité d'origines et d'approches, il résulte que les définitions et les pratiques de l'intertextualité sont également diverses; si la richesse du concept semble autoriser les interprétations les plus larges, un certain nombre d'études pourtant, cédant à la mode de l'intertextualité, semblent avoir un lien théorique beaucoup trop distendu avec le concept.

4 Le premier travers, repéré par la plupart des critiques, est de retomber, sous le nom nouveau d' " intertextualité », dans une vieille critique de sources ; cela consiste, dit Marc Angenot ${ }^{5}$, «à faire du neuf avec du vieux, et par exemple à appeler analyse intertextuelle, une bien traditionnelle critique philologique des sources et des influences littéraires ». Or l'intertextualité ne demande pas de "prouver le contact entre l'auteur et ses prédécesseurs. Il suffit pour qu'il y ait intertexte que le lecteur fasse nécessairement le rapprochement entre l'auteur et ses prédécesseurs $»^{6}$. Il revient au lecteur, par l'étendue de sa culture ${ }^{7}$, de déterminer la « dose » d'intertextualité du texte : seul récepteur (dans chaque acte de lecture) il est donc seul juge, seul à établir l'intertexte. Partant, se soulève le problème majeur de l'intertextualité : parce que le récepteur établit l'intertexte, cet intertexte peut varier d'un lecteur à l'autre, selon la culture et les lectures antérieures des lecteurs. Si la responsabilité du renvoi intertextuel incombe réellement au seul lecteur, l'intertextualité est-elle encore légitime ? Il est, en fait, impossible de décider du moment où l'intertexte est délibérément convoqué par l'auteur, inconsciemment provoqué par lui, ou redevable au lecteur seulement. Le passage de l'une à l'autre de ces catégories est évidemment graduel. On ne peut nier que l'intertextualité soit un phénomène de réception. C'est pourquoi il faut accepter ce sens très large de l'intertextualité, bien que le concept, probablement, risque alors de se désintégrer totalement.

5 Un autre élargissement fréquent du concept de texte, et par là d'intertexte, s'opère vers les domaines mythique et historique. En 1987, Marc Eigeldinger précisait à ce sujet: «Mon projet est de ne pas limiter la notion d'intertextualité à la seule littérature, mais de l'étendre aux divers domaines de la culture. Elle peut être liée à l'émergence d'un autre langage à l'intérieur du langage littéraire; par exemple celui des beaux-arts et de la musique, celui de la Bible ou de la mythologie, ainsi que celui de la philosophie $»^{8}$. De fait, ce serait donc maintenant toute allusion à notre culture qui relèverait de l'intertextualité. On est loin de la primitive de l'intertexte, qui renvoyait à une œuvre antérieure - c'est le cas de la Bible, mais non de toute la tradition mythologique. La notion d'intertextualité, de "relation entre différents textes écrits ", se dissout ainsi dans une définition plus large de simple référence culturelle ou artistique. L'intérêt de toute la réflexion - sur la productivité du texte, sur l'origine de l'écriture - qui accompagnait le concept d'intertextualité s'estompe, et n'est pas remplacé. Un renvoi à un texte écrit n'est pas la même chose qu'une référence culturelle. Respecter cette distinction éviterait une nouvelle dilution du concept.

6 Enfin, l'ultime support du renvoi intertextuel, problématique pour nous, est celui des genres. Confronter un texte à son code générique est très intéressant mais s'agit-il d'inter textualité ? Le genre n'existe pas sous forme de texte, il n'est qu'une abstraction, une émanation d'une multiplicité de textes. Sans doute sera-t-il préférable alors, sans nier 
l'intérêt et la richesse d'une telle étude, de lui donner le nom proposé par Genette, " architextualité ", d'étudier sous ce nom les rapports qu'un texte entretient avec le genre dont il se réclame ou qu'il parodie ${ }^{10}$, ou contre lequel il s'élève au contraire, et de réserver le nom d'intertextualité pour les rapports entre les textes, qui sont des réalisations uniques à l'intérieur d'un genre.

Dans la pratique concrète de tous les critiques, on constate deux tendances générales plutôt opposées. Certains, à l'instar de M. Riffaterre, s'attachent à élucider des allusions, des citations. Ils focalisent leur étude sur un vers, un mot, dont la dissonance avec le reste du texte nécessite le recours à un intertexte. Ils restent donc dans la microstructure. D'autres, sur le modèle de G. Genette, plutôt que d'étudier les microstructures, les fragments, privilégient les macrostructures: les structures, l'anecdote ou les traits génériques d'un texte. Laurent Jenny, réexaminant cette opposition, distingue bien entre l'intertextualité proprement dite, comme «rapports de texte à texte en tant qu'ensembles structurés »"11, et l'intertextualité faible - qui n'est pas vraiment, selon lui, de l'intertextualité -, faite d'allusions, de simples réminiscences: « chaque fois qu'il y a emprunt d'une unité textuelle abstraite de son contexte et insérée telle quelle dans un nouveau syntagme textuel, à titre d'élément paradigmatique $»^{12}$. Certainement, une importante différence - mais de degré, et non de nature - s'observe entre l'unique citation d'un texte, et la récriture de la structure entière ou quasi entière d'un texte. Le fonctionnement sera le même, mais le peu d'importance dans la structure et l'économie du nouveau texte d'une citation isolée peut mériter, effectivement, que l'on parle d'intertextualité faible.

8 L'idée d'intertextualité, affirme Marc Angenot ${ }^{13}$, «sert au telquelien ${ }^{14}$ à proclamer la bonne nouvelle de la mort du sujet ». Les textes étant dans un engendrement perpétuel, l'auteur comme sujet individuel, définition héritée de la tradition du XIX ${ }^{\text {ème }}$ siècle, n'a plus de crédibilité : à travers sa voix ce sont des dizaines, des centaines d'autres voix qui s'expriment, surtout dans le roman, genre polyphonique par excellence. « À la place de la notion d'intersubjectivité s'installe celle d'intertextualité » affirme Kristeva ${ }^{15}$ : ce sont les textes qui dialoguent, non les sujets, lesquels sont totalement expulsés des œuvres. Or, il nous semble que dans et grâce à l'intertextualité, le sujet ne meurt pas mais s'affirme. On peut donner l'exemple des citations de Proust dans La Bataille de Pharsale de Claude Simon. Mises en évidence comme la voix d'autrui par les italiques, ces citations collées prouvent l'hétérogénéité énonciative du texte. L'émotion de l'art, pour le lecteur, c'est alors cette double rencontre : celle de l'auteur qu'il lit, dont la voix lui parvient à travers ses mots, et celle, plus lointaine encore, de l'auteur avec lequel le livre dialogue. Si, faute des compétences culturelles nécessaires, le lecteur ne reconnaît pas la voix étrangère (dans notre exemple, celle de Proust), alors la mort du sujet devient effective : le lecteur ne perçoit qu'une voix, homogène et lisse, dans le texte.

9 Enfin, un des points les plus controversés est le problème de cette compétence culturelle du lecteur. On se souvient que M. Riffaterre s'est toujours tenu à une nette séparation entre l'intertextualité aléatoire et l'intertextualité obligatoire. L'intertextualité aléatoire, qu'il n'étudie pas, est fonction de la compétence du lecteur : selon sa culture, il verra, ou ne verra pas, les allusions et réminiscences d'autres textes se trouvant dans le texte qu'il lit. M. Riffaterre préfère se consacrer à l'intertextualité obligatoire, celle qui se présente sous forme d'agrammaticalités sémantiques, syntaxiques, morphologiques, et qui nécessite absolument le renvoi à l'intertexte, même si celui-ci n'est pas identifié par le lecteur. La plupart des critiques s'accorde généralement à dire que cette distinction entre 
les deux intertextualités ne tient pas. Peut-être s'agit-il d'une question de mots: M. Riffaterre n'aurait pas dû parler d'intertextualité aléatoire et obligatoire puisque, - et lui-même le reconnaît - l'intertextualité est toujours aléatoire, toujours fonction de la culture du lecteur. Il peut ne pas connaître l'intertexte, ni même sentir l'appel à l'intertexte. Il ne lui sera pas impossible de comprendre le texte.

À partir d'une définition assez large de l'intertextualité comme " croisement de textes », les théoriciens ont voulu se doter d'outils pratiques permettant d'étudier l'intertextualité ; dès lors, ils se sont heurtés à un grand nombre de questions, notamment celle de la nature du support du renvoi intertextuel, et celle de l'alternative entre microstructure et macrostructure. Plus délicat, s'est posé le problème de la compétence culturelle du récepteur. Les divergences d'opinion sur ces sujets et un certain flou conceptuel que l'on remarque dans les études intertextuelles n'ont pas aidé le concept à devenir un réel outil pratique et incontesté.

11 Finalement, il n'est resté de l'intertextualité qu'une définition très large, très théorique, dont on ne voit pas en quoi elle pourrait aider une poétique ou une stylistique des textes. Ainsi de l'expérience de Barthes :

«Lisant un texte rapporté par Stendhal (mais qui n'est pas de lui) j'y retrouve Proust par un détail minuscule [...]. Je comprends que l'œuvre de Proust est, du moins pour moi, l'œuvre de référence, la mathésis générale, le mandala de toute la cosmogonie littéraire [...]. Et c'est bien cela l'inter-texte : l'impossibilité de vivre hors du texte infini - que ce texte soit Proust, ou le journal quotidien, ou l'écran télévisuel : le livre fait le sens, le sens fait la vie $»^{16}$.

12 Ce sentiment de déjà-lu, l'idée de la bibliothèque universelle, s'ils sont incontestables, n'en sont pas moins décevants en stylistique. Le concept permet de mieux cerner la lecture et ses enjeux, mais il entre difficilement en jeu dans une description matérielle. À cause de ce flou, l'intertextualité, après avoir été à la mode pendant les années 70 et 80, a lassé peu à peu les critiques et les universitaires ${ }^{17}$. Les différents catalogues bibliographiques viennent confirmer cette impression de mode: après que de nombreuses études lui ont été consacrées depuis 1979, et de façon croissante jusqu'à la moitié des années 80 , l'intertextualité a vu sa popularité diminuer, et le nombre d'articles et d'ouvrages autour de ce concept a fortement décru depuis 1985.

\section{Les récritures}

13 Semblant succéder à l'intertextualité, un nouveau concept apparaît ou resurgit alors dans les années 80 , celui de "récriture » ou "réécriture ». Notre propos est d'utiliser cette alternative orthographique pour distinguer entre la "réécriture » génétique et la " récriture » intertextuelle. Là encore, des définitions nettes et rigoureuses s'imposent, pour éviter de retomber dans un autre flou, ou de seulement rebaptiser l'intertextualité.

iture ${ }^{18}$ ou réécriture est « l'action, le fait de réécrire », c'est-à-dire de " donner une nouvelle version d'un texte déjà écrit » ou de "réinventer, donner une nouvelle version de quelque chose $»^{19}$. Cet aspect mérite qu'on s'y attarde: "texte déjà écrit » peut signifier aussi bien « texte déjà publié » que « texte manuscrit ». Or, la démarche n'est pas la même dans les deux cas.

15 La critique génétique, depuis quelques années déjà, étudie les manuscrits et les avanttextes d'œuvres publiées ${ }^{20}$. La réécriture est alors la somme de préparations, de correc tions et de ratures, de variantes successives d'un même texte que l'auteur écrit - et que, la 
plupart du temps, il ne montre pas au lecteur. C'est dans cette acception que le terme de « réécriture » est le plus souvent pris. Pour cette raison, il serait préférable d'utiliser le terme de « récriture » avec un seul «é » pour désigner des transformations qui, même quand elles s'apparentent à celles de la réécriture génétique, en sont pourtant par essence différentes. En effet, il ne faut pas oublier que les avant-textes n'ont rien de commun, sociologiquement parlant, avec les livres publiés ${ }^{21}$. Le seul, l'authentique contact du lecteur ordinaire avec l'œuvre d'art littéraire se fait à travers le livre publié ; les manuscrits, eux ne sont livrés qu'à l'étude critique. Notre récriture, comme volonté manifeste d'un auteur de récrire le livre d'autrui ou de récrire un de ses propres livres déjà publiés, ou un de ses propres textes à l'intérieur d'un livre, n'est pas de la réécriture génétique.

La récriture, selon Georges Molinié22 , ne se pense qu'en termes fonctionnels : « elle définit une activité scripturaire qui s'établit forcément sur une corrélation suivie entre deux éléments. L'un de ces éléments est évidemment stable, qu'il s'agisse d'un discours littéraire de base, réalisé, sous la forme d'un texte, ou de tout un style (verrouillage figé de combinaisons stylématiques). L'autre élément peut être présenté comme l'écriture d'un nouveau texte, ou la mise en exercice d'un nouveau style ». Dans ces nouveaux textes interviennent des variantes, car la récriture n'est pas clonique, répétition totalement exacte.

Il est nécessaire de revenir sur certains aspects que la récriture et l'intertextualité ont ou n'ont pas en commun. Définir la récriture sur l'horizon de l'intertextualité, permet de démontrer l'efficacité du concept de récriture en stylistique.

Critère problématique par la difficulté de sa mesure, l'ampleur ou la fréquence du phénomène est primordiale pour distinguer entre l'intertextualité et la récriture. Laurent Jenny séparait l'intertextualité faible, simple allusion ou réminiscence, de l'intertextualité proprement dite, comme rapport de texte à texte en tant qu'ensembles structurés. Ce critère semble effectivement séparer deux concepts différents. Une simple allusion ou réminiscence dans l'esprit du lecteur relève bien sûr de l'intertextualité, et non de la récriture. Celle-ci nécessite tout un ensemble de marques matérielles, tangibles et probantes: pas une unique lexie, ni quelques lettres, comme c'est le cas dans les paragrammes. Ces marques concrètes, que l'on est tout logiquement appelé à nommer des récritures, - en opposant la récriture, le concept, aux récritures comme marques concrètes -, doivent former un ensemble s'étendant tout au long d'un texte. Il s'agit, ici aussi, d'intertextualité - on peut parler de récriture intertextuelle - : mais si la récriture d'autrui présuppose l'intertextualité, l'intertextualité, en revanche, ne présuppose pas la récriture. Une citation, par exemple, isolée dans un texte, représente un fait d'inter textualité et non pas une récriture. On conviendra de ne nommer " récriture » que des faisceaux suffisamment importants de marques concrètes et probantes.

R. Barthes, dans la définition très large qu'il donnait de l'intertextualité, expliquait combien la lecture d'une phrase rapportée par Flaubert lui rappelait un fragment de Proust. Effectivement, quand on prend l'intertextualité dans son sens le plus large, on constate qu'à tout moment, en lisant un texte, des souvenirs textuels affluent à notre mémoire, que nous seuls, peut-être, mettons dans ce texte. Si l'on prend une définition plus restreinte de l'intertextualité, en refusant au récepteur ce rôle omnipotent, et en rendant à l'auteur une place, les réminiscences intertextuelles que l'auteur inscrit dans son texte peuvent être fortuites et non-intentionnelles. Peut-être une imprégnation forte d'un texte pourrait-elle provoquer une récriture formellement exacte; la récriture se 
différencierait alors de l'intertextualité uniquement par son caractère formel. Mais dans la plupart des cas, la récriture présuppose et exige une intention de récrire. Elle se situe alors autant du côté de la production que de la réception. Qu'il s'agisse de plagiat, d'imitation ou de récriture, l'auteur a bien entendu agi en toute conscience et volontairement - alors que l'intertextualité peut être involontaire - ; il a copié ou modifié le livre-source, ouvert à côté de lui. L'ampleur de la récriture, le nombre important de récritures suffisent à apporter au critique la preuve formelle de l'intentionnalité de récrire de l'auteur. Certes, le critique est seul juge de cette ampleur, mais son interprétation reste beaucoup moins aléatoire que dans le cas de l'intertextualité.

L'intertextualité comme la récriture ont en commun d'accorder en même temps la part belle à la réception. La lecture est une interaction entre le texte et son lecteur. L'enracinement dans le contexte historique, social et culturel du lecteur, influe sur sa lecture; l'interprétation d'un texte varie donc avec chaque lecteur. Le lecteur est l'implicite du texte, une donnée nécessaire et inévitable pour la lecture d'un texte ${ }^{23}$. L'intertextualité reste bien souvent aléatoire: elle dépend de chaque lecteur, et de sa culture. Elle peut aussi bien n'être pas perçue, ou encore le lecteur est susceptible de la projeter dans tel texte, qu'un autre lecteur jugera dépourvu d'intertextualité. La récriture, toujours grâce à son ampleur, est obligatoire; non dans le sens où elle est forcément perçue mais dans le sens où, même si elle n'est pas affichée, une fois démontrée, elle est incontestable et sans appel.

21 Dans cet esprit, une autre conséquence s'impose : alors que l'intertextualité méprise la chronologie ${ }^{24}$ (Flaubert, dans l'exemple de Barthes, appelant Proust), et que l'on peut lire Edipe roi, par exemple, en songeant à Freud, la récriture, du fait de son intentionnalité, est forcément récriture d'un texte antérieur. Nous restreignons ainsi le sens de « récriture », en laissant à « intertextualité » le plus de liberté possible. Le concept, pour être pratique, exige des limites précises. Cette position laisse moins de place aux délires interprétatifs de certains, qui confondent les ressemblances génériques et sociologiques avec l'intertextualité, ou qui érigent leurs moindres réminiscences en intertextualité.

Enfin, une allusion intertextuelle peut référer aux différents systèmes sémiotiques: la peinture, la musique... Cela passe bien sûr par une verbalisation. Le tableau, le morceau de musique est décrit, évoqué au travers de mots. Depuis toujours on rencontre dans la littérature ces échanges "intersémiotiques ». On considérera donc la récriture comme une restriction de ces pratiques à un seul système sémiotique : le langage verbal. Récrire, en effet, c'est écrire quelque chose qui a déjà été écrit, du déjà-verbal.

L'intertextualité, et il faudrait s'en tenir à cette définition si l'on veut bien distinguer les deux concepts, est une utilisation des œuvres d'autrui : un rapport entre des textes distincts, différents. Certains nomment cependant «intertextualité restreinte ${ }^{25}$ les rapports entre textes d'un même auteur, par exemple entre Jean Santeuil et À la recherche du temps perdu de Proust... Or il s'agit là plutôt d'une récriture, plus proche de la réécriture génétique que d'allusions ou de citations intertextuelles. Il vaudrait donc mieux parler de récriture dans l'exemple évoqué.

La récriture possède différentes facettes. La récriture qui s'impose généralement et naturellement à l'esprit est la récriture d'autrui, la récriture proprement intertextuelle, comme par exemple Ulysse de James Joyce sur les traces de son célèbre prédécesseur grec, Homère. D'autres types de récriture fonctionnent de façon tout à fait similaire à la récriture intertextuelle. Ce sont toutes les récritures de soi, qu'il s'agisse de récritures d'un livre à un autre ou encore à l'intérieur d'un même livre, d'un chapitre à l'autre. Dans 
tous les cas, les mécanismes de répétition et d'indexation sont les mêmes, et justifient l'appellation générique de « récriture ».

De l'intertextualité à la récriture : ce n'est surtout pas le parcours d'un concept à son synonyme. Certains critiques se sont contentés malheureusement de rebaptiser du nom de récriture le concept d'intertextualité. Les deux concepts ne sont pas synonymes, et la notion de récriture en stylistique doit reposer sur des fondements solides. La récriture est un concept nécessaire, essentiel pour comprendre certaines œuvres entièrement fondées sur elle ${ }^{26}$, puisqu'elle se caractérise par une ampleur, une monumentalité qui affecte tout un roman.

D'autre part, le concept n'est pas aléatoire : alors que l'intertextualité fait plonger parfois la critique dans des interprétations hasardeuses ou abusives. En effet, une étude de récriture peut s'appuyer sur une stylistique pratique et une étude précise du texte. Un faisceau de preuves verbales, reposant toutes sur la répétition, touchant aussi bien les signifiants que les signifiés, permet de rejeter, le plus possible, l'aléatoire, sans nier pour autant l'importance du rôle du lecteur dans la réception d'une œuvre littéraire en général, et d'une œuvre fondée sur la récriture plus particulièrement.

\section{NOTES}

1. Par exemple Nathalie Piégay-Gros, Introduction à l'intertextualité, Dunod, 1996.

2. Semeiotikè, recherches pour une sémanalyse, Paris, Seuil, 1969, p. 145.

3. «La stratégie de la forme », Poétique $\mathrm{n}^{\circ} 27,1976$, p. 257-281.

4. Palimpsestes, la littérature au second degré, Seuil, 1982.

5. «L'intertextualité : enquête sur l'émergence et la diffusion d'un champ notionnel », Revue des sciences humaines tome LX n 189, janvier-mars 1983, p. 121-135.

6. Michael Riffaterre, «Sémiotique intertextuelle : l'interprétant », Revue d'esthétique $\mathrm{n}^{\circ}$ 1-2, 1979, p. 131.

7. Quand bien même elle n'atteint pas l'érudition gigantesque de Michael Riffaterre, laquelle n'est pas à la portée de tous, comme le souligne Uri Eisenzweig : « un concept plein d'intérêts ", Texte $\mathrm{n}^{\circ} 2,1983$, p. 160-170.

8. Mythologie et intertextualité, Genève, Slatkine, 1987, p. 15.

9. «Prétendre [...] qu'il y a de l'intertextualité entre divers textes-occurrences, alors qu'il s'agit seulement de structures sémantiques et/ou syntaxiques communes à un type (ou à un "genre ») de discours, revient à nier l'existence des discours sociaux (et des sémiotiques transcendant la communication interindividuelle) », A.-J. Greimas, Joseph Courtès, Sémiotique. Dictionnaire raisonné de la théorie du langage, Paris, Hachette, 1993.

10. $Y$ compris pour les textes du XXème siècle qui s'affichent comme des parodies, des mixtes ou des refus de genres, et restent donc toujours appréhendables en fonction du concept de genre.

11. Art. cit., p. 263.

12. Art. cit., p. 262.

13. Art. cit., p. 125.

14. C'est-à-dire aux collaborateurs de Tel quel.

15. Semeiotikè, op. cit., p. 146. 
16. Roland Barthes, Le Plaisir du texte, Seuil, 1993, p. 58-59.

17. Marc Angenot (art. cit., p. 122) parle de «mode intellectuelle».

18. Le verbe " récrire » est attesté mais le mot « récriture » n'existe pas dans les dictionnaires Robert, Larousse et le Trésor de la langue française.

19. Trésor de la langue française, Gallimard, 1990.

20. Voir par exemple L'Écriture et ses doubles. Genèse et variation textuelle, Daniel Ferrer et alii., éd. du C.N.R.S., Paris, 1991, ou Claudette Oriol-Boyer : La Réécriture, Grenoble, Céditel, 1990.

21. Il faut reconnaître pourtant qu'ils ont tendance à investir certaines éditions, telles celles de la Pléiade. Le lecteur est placé de fait devant la reproduction des manuscrits ou des corrections. Cette volonté de retrouver les archives, de transformer la lecture en recherche archéologique et génétique est-elle réellement fondée?

22. "Les lieux du discours littéraire", Lieux communs. Topoï, stéréotypes, clichés, dir. Christian Plantin, Paris, éd. Kimé, 1993, p. 92-100.

23. Wolfgang Iser a exposé les fondements de cette esthétique de la réception dans L'Acte de lecture, théorie de l'effet esthétique, Bruxelles, Mardaga, 1976.

24. "On peut être influencé par un livre que l'on a convenablement oublié ou retrouver, d'une culture ancienne dont on s'est abondamment nourri, un précieux linéament ; on peut subir l'impact de ce qu'on n'a pas lu, ou peut, enfin, retrouver ce que l'on n'a jamais su, reconnaître ce que l'on n'a jamais vu, et reproduire ce que l'on n'a jamais lu. » Michel Lafon, Borges ou la réécriture, Seuil, 1990, p. 37.

25. «Le texte n'émane pas, isolément, d'un auteur, en tant que stade actuel au cours des relatifs changements de sa vie. Il s'écrit résolument en relation avec les textes déjà écrits par le même signataire ». Jean Ricardou, «Terrorisme, théorie », p. 12-13, in Robbe-Grillet, colloque de Cerisy, U.G.E., 10/18, 1976.

26. Nous pensons bien sûr au Nouveau Roman.

\section{RÉSUMÉS}

Le propos de cet article est d'éclairer le concept de récriture en le confrontant d'une part à celui d'intertextualité, du point de vue de la réception, et d'autre part, du point de vue de la production, à celui de réécriture, utilisé dans la critique génétique. Le concept de récriture se situe ainsi aux confins d'une étude de la production et de la réception. La récriture confirme le rôle actif du lecteur, mais se donne une vérification stylistique concrète en s'affirmant comme intentionnelle et massive (étendue à tout un livre). Définir la récriture sur l'horizon de l'intertextualité, permet ainsi de montrer l'efficacité du concept de récriture en stylistique.

\section{AUTEUR}

\section{ANNE-CLAIRE GIGNOUX}

Université de Paris IV-Sorbonne 